Cita bibliográfica: Carrillo Sánchez, L. G., García Calderón, C. I., Cuevas Contreras, T. y Ruiz León, A. A. (2020). Big data en la marca Ciudad de México ante la fragmentación de audiencias. Investigaciones Turísticas (20), pp. 124-142. https://doi.org/10.14198/INTURI2020.20.06

\title{
Big data en la marca Ciudad de México ante la fragmentación de audiencias
}

\section{Big data in the Mexico City brand in the context of audience fragmentation}

\author{
Laura Grisel Carrillo Sánchez (iD, Universidad Nacional Autónoma de México, México \\ saselgri@hotmail.com
}
Carola Isabel García Calderón iD, Universidad Nacional Autónoma de México, México carolinacreel@hotmail.com

Tomás Cuevas Contreras (iD, Universidad Autónoma de Ciudad Juárez, México tcuevas@uaci.mx

\begin{abstract}
Alejandro Arnulfo Ruiz León (iD, Universidad Nacional Autónoma de México, México rarnulfo@gmail.com
\end{abstract}

\section{RESUMEN}

El artículo analiza comportamiento coyuntural en el ámbito mundial, ante el esfuerzo mediático del destino turístico por los mercados dispersos. El Internet ha influenciado la comunicación e impulsando las nuevas tecnologías, las cuales han avanzado vertiginosamente en los primeros tres lustros del siglo XXI, esto ha impactado a las diversas actividades económicas, entre las que destaca el turismo. En consecuencia, el consumidor, ha dejado de tener un papel receptivo, para ser un decisor del material mediático que consume, además de contar con la oportunidad de ser también un proveedor de información. La Ciudad de México (CDMX), como segmento de turismo, no es la excepción, la misma depende de la comunicación de actores públicos y privados, los cuales, aunque poseen determinadas metodologías y herramientas para acceder a sus públicos, se encuentran con una audiencia que decide sobre lo que recibe y opina en la web, con ello, el objetivo de la investigación es referente a la valoración de los conceptos asociados, páginas y organismos relacionados con el turismo de la CDMX en la web entre la audiencia nacional e internacional, originado tanto por los esfuerzos de gobierno y empresarios, como de la audiencia que también ya es una proveedora de información. Asimismo, a través de la investigación se busca conocer a los países con el mayor interés en el turismo de la Ciudad de México, ya que ello puede resultar de gran importancia para direccionar las estrategias mercadológicas. 
La metodología empleada para la exploración del fenómeno es de tipo documental y cuantitativa, ya que en una primera etapa incluye el análisis documental y posteriormente hace la exploración matemática a través del empleo del análisis de redes (ARS) para valorar el papel del Big data y Smart Data en la marca CDMX ante la fragmentación de audiencias.

Palabras clave: Big Data; Smart Data; Marca Ciudad; Ciudad de México (CDMX); fragmentación de audiencias.

\section{ABSTRACT}

The article analyzes economic behavior throughout the world in response to the media effort of tourist destinations in dispersed markets. The Internet has influenced communication and boosted new technologies, which have advanced dramatically in the first three decades of the twenty-first century. This has had an impact on the various economic activities, among which tourism is particularly salient. Consequently, consumers no longer have a receptive role. They have become decision makers with respect to the media material that they consume in addition to having the opportunity of being information providers. Mexico City (CDMX), as a tourism segment, is no exception. It depends on the communication of public and private actors, who, while having certain methodologies and tools to access their audiences, interact with an audience that decides on what it receives and gives its opinion on the web. Therefore, the objective of the research is to assess the associated concepts, pages and organizations related to tourism in CDMX on the website aimed at both a domestic and international audience, designed by the government and private sector together with the input of the audience as an information provider. Likewise, the study seeks to determine the countries with the greatest interest in Mexico City as a tourist destination, since this can be highly important for directing the marketing strategies.

The methodology used to explore the phenomenon takes a documentary and quantitative form, since in a first stage it includes the documentary analysis and subsequently makes the mathematical exploration through the use of network analysis (ARS) to assess the role of Big data and Smart Data in the CDMX brand before the fragmentation of audiences.

Keywords: BIG DATA; Smart Data; City Brand; Mexico City (CDMX); audience fragmentation.

\section{INTRODUCCIÓN}

La comunicación es una disciplina que ha actuado en paralelo al desarrollo de las sociedades y por ende de las organizaciones, ya que gracias a esta se han podido poner en común ideas y conceptos que permiten la sinergia social que hace posible al ser humano vivir en comunidad. Específicamente dentro del concepto de organización definido como un "conjunto de personas constituido de forma deliberada para cumplir un propósito específico" (Robbins y Coulter, 2014, p.7), la comunicación resulta fundamental dado que la organización tiene procesos de relación en el interior y en el exterior, lo que demanda de mensajes bien segmentados acorde a sus audiencias y necesidades específicas de la organización. 
La aplicación de la comunicación en la actividad turística es muy compleja, de inicio debe tomarse en cuenta que el turismo tiene una gran relevancia en la economía internacional, aunado a ello

"existe un amplio consenso sobre el efecto positivo que el turismo internacional tiene sobre el crecimiento económico de un destino en el largo plazo. La transmisión de este efecto se da a través de distintos canales como la provisión de divisas, el estímulo de la inversión en infraestructura, del capital humano, y la competencia, la generación de empleo y el incremento de los ingresos, y el aprovechamiento de economías de escala y de alcance" (Brida, Lanzilotta, Pereyra y Pizzolon, 2013, p. 77).

En el caso mexicano el turismo es una actividad económica muy relevante dada su aportación a la economía, de acuerdo con la World Tourism Organization (UNWTO), el país se coloca en el lugar 16 por ingreso de divisas durante 2018 con 22.5 mil millones de dólares y en lugar 7 en llegada de visitantes con 41.4 millones de turistas.

En este ambiente tan complejo en el que se desenvuelve la actividad, resulta vital la evaluación de la comunicación, dado que la misma cada vez demanda un enfoque mayor, los públicos tienden a tener un consumo más específico y aunque se sigue bombardeando con una gran cantidad de impactos, derivado de "ciertos avances experimentados de internet, como el uso de un mayor ancho de banda y protocolos comunes para el flujo de datos entre usuarios" (Jova, Bradshaw y Despaigne, 2015, p. 2), se está permitiendo una evolución hacia un consumo muy segmentado. Es por ello que las actividades comunicativas demandan también una alta segmentación que permita un abordaje estratégico a sus públicos, a través del cual las organizaciones (tanto a gobiernos, como a empresas) pueden llegar de forma atinada y con mensajes puntuales a aquellos que buscan impactar. No obstante, este proceso no es sencillo, dada la complejidad de la comunicación y la gran cantidad de medios existentes.

En México se busca abordar tanto al público nacional como al internacional en cuestiones de turismo, sin embargo, para fines de este estudio se analiza el enfoque en el mercado internacional, dado que este último es de gran importancia para el país, por la posibilidad de ingresos de divisas que trae consigo, lo cual puede beneficiar la balanza comercial nacional. Ante este escenario el Big data, entendido como una "estrategia organizacional, tecnológica y táctica que facilita capturar, almacenar, procesar y analizar los grandes volúmenes de datos generados en toda la cadena de valor de la empresa, que varía según el sector, la industria y las necesidades de cada compañía" (Ortiz, Joyanes y Giraldo, 2016: 8), cobra una gran relevancia, ya que este permite gestionar una gran cantidad de datos para la toma de decisiones en las organizaciones y el logro del enfoque de las campañas digitales. Es preciso considerar que el acceso a la información de estas bases no es sencillo dado no solo el alto costo de las mismas, sino un conocimiento complejo para su manejo. "El análisis de datos se realiza con tecnologías de bases de datos como NoSQL, Hadoop y MapReduce, las cuales soportan el procesamiento del Big Data" (Camargo-Vega; Camargo-Ortega y Joyanes, 2015, p.67).

Con base en este contexto surgen plataformas gratuitas como Google trends o de paga como Sem Rush o Similar Web, las cuales aunque con costo, permiten a través de un manejo 
más amigable la obtención de datos de grandes volúmenes de información, los cuales hacen referencia al Big data, sin embargo a través del procesamiento de estas plataformas se entregan al usuario final de forma más simple para dar paso a lo que se le denomina Smart Data, obteniendo de esos grandes volúmenes de información, los datos clave para la gestión de las organizaciones y su gestión publicitaria, de acuerdo a la Universidad de Valencia se dice que el Big data se refiere a una gran cantidad de datos, mientras el Smart data, se refiere a tener un sistema que entrega al usuario los datos analizados. Ante ello el objetivo de la investigación es referente a la valoración de la relevancia de los conceptos asociados, páginas y organismos relacionados con el turismo de la CDMX en la web entre la audiencia nacional e internacional. Asimismo, a través de la investigación se busca conocer a los países con el mayor interés en el turismo de la Ciudad de México.

Por ello, la metodología empleada para la exploración incluye el análisis documental de diversas fuentes de información, la revisión del empleo del Big Data y el Smart Data, para ubicar el desempeño de términos relacionados con el turismo, la marca Ciudad de México y algunas páginas relacionadas con la actividad, para analizar su manejo en las redes, en el último semestre del 2017 y el primer semestre de 2018. Los resultados se plasman y analizan a través de redes para valorar el papel del Big data ante la fragmentación de audiencias, midiendo centralidad y de esta forma identificar en donde se están concentrando los grandes esfuerzos e intereses. El resultado es la síntesis y conclusiones con relación a la temática.

\section{DISCUSIÓN CONCEPTUAL}

El turismo es una actividad preponderante para el desarrollo de las economías de los países, este "se configura como uno de los principales sectores económicos a nivel internacional, adquiriendo importancia en los países en vías de desarrollo" (Orgaz y Moral, 2016, p. 1), dados los múltiples beneficiosos que puede tener para la economía local. Esto se da en gran parte a que el turismo implica muchas actividades, ya que los productos turísticos se conforman por "bienes y servicios a los turistas, incluyendo a los servicios de entretenimiento y de recreación u ocio, así como a todo activo natural, cultural y social donde se desarrolla gran parte de las actividades de servicio, que constituye, en muchos casos, el mayor atractivo para el turismo" (Morillo, 2011, p. 152).

Aunado a ello, de acuerdo a Datatur durante 2018, la llegada de turistas ascendió a 41.4 millones, lo cual fue superior en 2 millones 156 mil turistas a lo observado en 2017 y con un crecimiento anual del $5.5 \%$, esto es favorecedor, dada la gran diversidad de satisfactores que pueden ofrecerse al mercado, lo cual puede ir desde sencillas actividades ecoturísticas, hasta una gran infraestructura hotelera capaz de atender a segmentos económicos privilegiados- Aunque"[...] México no ha logrado ubicarse dentro de los primeros cinco principales destinos mundiales, a pesar de la multiplicidad de atractivos turísticos y a la voluntad política del pasado" (Cuevas, Zizaldra y Carrillo, 2017, p.186).

Sin embargo, aunque la actividad ha ido al alza, es importante destacar las ventajas competitivas de los productos, ya que hay una gran cantidad de oferentes en el mercado. Aunque:" [...] ventaja competitiva es un concepto más empresarial que económico y hace 
referencia a un instrumento activo o a un proceso dinámico de acumulación de factores internos y externos" (Sobrino, 2002, p. 318), desde la óptica capitalista para los gobiernos e incluso para las empresas relacionadas con el turismo tiene una gran importancia, ya que el que se logre la venta de un destino requiere de la suma de factores del producto como tal, por ejemplo la calidad de un hotel, las instalaciones y el servicio.

El producto turístico es muy complejo y hay otras variables que están no solo en el diseño que los empresarios puedan hacer con el mismo, sino de otros factores inherentes al destino considerando aspectos como la imagen y la afectación que tenga la misma por cuestiones como la delincuencia, la corrupción y otros problemas que puedan tenerse de conectividad o seguridad en general.

En este contexto la Ciudad de México no es la excepción, aunque existen grandes esfuerzos gubernamentales por mostrar las magnas cualidades de la CDMX y además se busca hacerla destacar como un destino cosmopolita, la realidad es que están presentes en su trama problemas económicos, culturales y sociales. Estas dificultades, restan en vez de apuntalar al discurso oficial para atraer visitantes. Inseguridad, manifestaciones y contaminación son algunos de los puntos que pueden afectar la imagen del destino. Es una tarea que debe reforzarse de forma integral, para ofrecer un destino con mayores y mejores atributos para los visitantes.

Con respecto a lo antes planteado, México como país, es un destino con grandes atractivos turísticos, los mismos son principalmente enmarcados en el segmento sol y playa, por lo que las ciudades tienen mucho por hacer en este sentido. El desarrollo del turismo de ciudades puede explotar el turismo de ocio aprovechando parques, restaurantes y espectáculos, e incluso otros tipos de turismo que pueden darse dentro de la ciudad como lo es el turismo gay o el médico.

Debe considerarse que si bien el turismo casi siempre es visto bajo la óptica de esparcimiento, el mismo por definición se concibe de acuerdo a la UNWTO como "un fenómeno de carácter cultural, social y económico relacionado con el desplazamiento de los individuos a lugares que están fuera de su lugar de residencia habitual por asuntos personales, profesionales o de negocios", de tal forma puede haber otros enfoques de turismo como el médico, que da grandes posibilidades de desarrollo para las ciudades.

Sin embargo, aún falta mucho para explotar esta vertiente, ya que incluso a nivel de hospitales hay poca explotación del concepto, siendo pocas opciones en las que se correlacionan la oferta médica con los servicios necesarios de hotelería. Por ejemplo, en la Ciudad de México con base en datos del INEGI, en el 2018 se tenían registradas 260 unidades médicas de las cuales no hay un registro en esta base de cuales ofrecen servicios de hotelería, este concepto puede ser ampliado a otros hospitales, con lo cual es factible enriquecer la oferta al momento que se apoya al turismo y se venden servicios complementarios al producto principal.

Asimismo, debe considerarse que la capital tiene productos relevantes, desde el punto de vista del placer y el ocio, plasmado en atractivos que van desde el enfoque gastronómico, museos, espectáculos, plazas, templos, etc. Aunado a ello es factible desarrollar eventos como congresos y convenciones dada la infraestructura existente. Pueden también aprovecharse 
aspectos como las diversas rutas aéreas que deben hacer escala en la Ciudad y la cercanía de lugares atractivos por su renombre internacional para el turismo como lo es Teotihuacán y ciertos lugares típicos del estado de Morelos.

En esta búsqueda hay diversos organismos clave que participan desde el enfoque gubernamental para el desarrollo de acciones dirigidas al desarrollo de la ciudad como lo es la Secretaría de Turismo Federal (SECTUR Federal), la Secretaría de Turismo de la Ciudad de México (SECTUR CDMX), el Consejo de Promoción Turística (CPTM) y el Fondo Mixto de Promoción Turística (FMPT). Cada uno de estos organismos busca acercarse de diversas formas a sus públicos y con ello de atraer a los mismos a visitar destinos mexicanos entre los que se encuentra la Ciudad de México.

SECTUR Federal de acuerdo con la Ley General de Turismo es la dependencia que realiza el diseño y guía de la política turística nacional, de tal forma la misma está encargada de la promoción de la actividad turística a nivel nacional e internacional en colaboración con el Consejo de Promoción, a través de lo cual se desarrollan acciones federales y estatales en búsqueda del desarrollo turístico. Esta dependencia revisa lo relacionado a la infraestructura, promoción, acuerdos y cooperación entre los diferentes actores involucrados en la actividad.

Respecto al CPTM, con base en la Ley General de Turismo, este organismo se encarga de coordinar, diseñar y operar estrategias promocionales a nivel nacional e internacional. A través de esta dependencia se consolidan proyectos y estrategias a mediano plazo, considerando con ello un escenario global que cada vez implica más retos y desarrollo de ventajas competitivas

Sectur de la CDMX, de acuerdo con la Ley de Turismo del Distrito Federal, es el organismo encargado de gestionar la política turística, con lo que busca impulsar el desarrollo turístico en Ciudad de México. Tiene un interés en la sustentabilidad, por lo que se enfoca en gran medida al medio ambiente. Asimismo, se inclina en la búsqueda de autenticidad sociocultural y el desarrollo de actividades que apoyen la economía por medio del empleo y cuestiones sociales.

Como último organismo clave se encuentra el FMPT, el cual, con base en la Ley de Turismo del Distrito Federal, es un fideicomiso público que se encarga de implementar, asesorar y financiar planes, programas y acciones de promoción en cuestiones turísticas. A través de esta dependencia también se busca fomentar la imagen de la Ciudad de México, por medio de campañas de promoción y publicidad nacional e internacional.

Es importante en el marco de la comunicación destacar los esfuerzos que se realizan por parte de todas estas organizaciones, considerando que esta es un punto clave para el posicionamiento de las mismas. "La comunicación supone diferenciación de la competencia y optimización de las relaciones con los distintos públicos con los que la organización convive" (Gómez y Benito, 2014, p.3). Los esfuerzos que estas dependencias realizan pueden ser percibidos por distintos individuos en el mundo, lo cual muestra el enfoque de estos y al mismo tiempo el interés de los públicos en el destino turístico. 
Derivado de que las pautas comunicativas llegan a diversos lugares del mundo debe considerarse que "el proceso comunicativo organizacional debe ser estructurado dentro de un sistema de significados que sean entendibles para todos, en donde se establezcan patrones del lenguaje que expresen claramente sus metas, sus espacios de información o reflexión y sus protocolos para la creación artística y de conocimiento, evitando así la construcción individual de metalenguajes por parte de los miembros del grupo laboral" (Zuñiga, 2010, p.75).

De ahí la importancia de que se logre la homogeneización hacia el interior de las organizaciones y que esto se observe en la comunicación externa que se lleva a cabo. Si bien existen eventos, ferias y esfuerzos de otra naturaleza comunicativa, lo importante es que se mantengan mensajes y esfuerzos afines en las distintas vertientes de comunicación dependiendo de los segmentos a los que se está llegando en el mercado.

Aunado a ello es importante considerar las grandes acepciones que se pueden dar a la comunicación como tal e incluso a la comunicación organizacional, por ejemplo, respecto a esta última algunos autores consideran a esta un sistema y por ende "un conjunto de recursos disponibles, prácticas, procesos y entornos para lograr el propósito común de la organización y hacer llegar los mensajes corporativos a todos los públicos de interés para la empresa y con objetivos adecuados a cada audiencia." (Méndez, 2007, p. 2).

De tal forma un punto que se reitera en estas definiciones es la gran importancia de lograr la vinculación entre los actores, lo cual puede realizarse a través de diversas herramientas como la publicidad, con lo cual se pueden lograr los objetivos que se tienen con los diferentes segmentos en quienes están interesados.

"En la actualidad, la publicidad se refiere a la manifestación de ideas, conceptos y valores asociados a productos servicios o marcas, con fines comerciales, siendo su objetivo construir, dirigir o transformar actitudes en sentidos que favorezca a intereses de empresas patrocinadoras o interesadas. Es decir, se apoya en un mensaje que se sostiene en el convencimiento, y no es la dialogicidad. El objetivo de la publicidad es la divulgación que tiene la compra como consecuencia" (Amon, Arcides, Pedrinho y Veríssimo, 2014, p.37 - 38).

La comunicación publicitaria dentro de las organizaciones es un punto clave para el posicionamiento de sus productos y/o servicios, con lo cual es posible lograr una convergencia entre la comunicación en el sentido de que esta debe estar bien comunicada con sus públicos entre los que se encuentra su audiencia como tal, además de la gran importancia de tener claro lo que se busca lograr con la misma.

En la actualidad la cantidad de información que llega a las audiencias es enorme, por lo que resalta la importancia del empleo adecuado del Big data, este término "se ha convertido en una tendencia a nivel mundial y aunque aún no cuenta con un concepto científico o académico consensuado, se augura cada día mayor crecimiento del mercado que lo envuelve y de las áreas de investigación asociadas" (Hernández, Duque y Moreno, 2017, p.2). De tal forma el término Big data implica "una nueva forma de gestionar el alto nivel de datos que existen y que se generan a nivel global en la actualidad y aprovecharlos en función de lograr las metas 
que se trazan las distintas empresas y organizaciones de diferentes sectores" (Plasencia, 2017, p.567)

Esto es fundamental, ya que resulta de suma importancia el aprovechamiento de estos datos que existen ante audiencias tan diversas y con consumos tan específicos. Asimismo debe considerarse que el uso de internet está en constante crecimiento, de acuerdo con la firma Hootsuite en un estudio realizado el primer semestre de 2018 , el $65 \%$ de la población mexicana son usuarios de internet (Hootsuite, Digital in 2018, p.31), y aunque no todos tienen el mismo rol, el cual puede ir desde un simple usuario hasta desempeñar una función como influencer, es notoria la gran penetración que se está teniendo en este sentido y la importancia que debe darse a ello.

Debe profundizarse, "el Big - Data y con distintas herramientas las organizaciones pueden medir y saber precisamente de una forma radica lo que está sucediendo en sus negocios y traducir directamente ese conocimiento en una toma de decisiones mejorada y en un rendimiento superior" (Goyzueta, 2015, p.148). No obstante, es importante considerar que el gran volumen de datos en ocasiones dificulta la integración de estos, de forma que realmente los mismos sirvan para la toma de decisiones y/o la actuación estratégica de las organizaciones. El análisis de datos requiere tecnologías sofisticadas, lo cual reduce las posibilidades de las empresas sobre todo las que se encuentran en el rubro de las PyMES para aplicar esta información.

Es necesario que en este gran volumen de datos las organizaciones distingan aquellos que sean relevantes para ellos, de manera que al reconocer los objetivos que se persiguen logren un análisis de la información que los lleve a generar valor a partir de estos datos, al tener una adecuada selección y con ello a lograr una determinación y visualización de la información que se traduzca en un diagnóstico de lo que se requiere y una base de partida para el diseño de estrategias.

Diversas empresas han visto en estos datos una oportunidad y han creado un nuevo concepto denominado Smart Data. Así, mientras en el Big Data se atienden cuestiones relacionadas con las seis V "volumen, velocidad, variedad veracidad, valor y visualización" (Ortiz, Aguilar y Giraldo, 2015, p.1), en este último concepto únicamente se centra en la veracidad y en el valor, con la finalidad de aplicar estos últimos a partir de sistemas de inteligencia artificial, por medio de los cuales se puedan tomar decisiones clave en las organizaciones, con ello se simplifican los datos de Big Data a través de diversas tecnologías, llegando al dato clave para las organizaciones.

Sin embargo, lo importante de ese análisis como en cualquier proceso de investigación es lograr la claridad del objetivo y a partir de ello obtener la información necesaria que lleve a obtener los datos que requieren las organizaciones. Ante la gran cantidad de información existen herramientas que entregan un procesamiento de estos datos y los entregan al usuario, logrando hacer este proceso más amigable, accesible y útil. 


\section{METODOLOGÍA}

Una vez desarrollada la investigación conceptual de forma documental y analizando la importancia de los datos con relación al Big Data, su relación con el Smart Data y el manejo de la comunicación en torno al turismo, se explica la forma en que se realiza el manejo de la información y la contrastación de los datos (Figura 1), esto para cumplir con el objetivo de la investigación el cual es referente a la valoración de los conceptos asociados, páginas y organismos relacionados con el turismo de la CDMX en la web entre la audiencia nacional e internacional, originados a partir de los actores gobierno, empresarios y audiencia. Asimismo, a través de la investigación se busca conocer a los países con el mayor interés en el turismo de la Ciudad de México, ya que ello puede resultar de gran importancia para direccionar las estrategias mercadológicas.

Se analizan los organismos que a nivel gubernamental son más importantes para el desarrollo de la actividad turística de la Ciudad de México. Los mismos son FMPT, SECTUR Federal, SECTUR CDMX y CPTM, de acuerdo con la información gubernamental analizada que corresponde a la Ley Federal de Turismo y la información interna de estos organismos.

Esto se realiza a través de las herramientas Google trends, SEM rush y Pro similar web, ya que las mismas tienen la posibilidad de procesar una gran cantidad de información relacionada con organismos, términos clave, páginas más visitadas y países, lo cual permite determinar la forma en la que se está percibiendo al destino mexicano al obtener la información más relevante en este sentido.

Con base en datos cuantitativos a través de Google Trends se analizó a los cinco países que estaban más interesados en estos términos en la WEB y se asignó un porcentaje de búsquedas. En algunos casos la búsqueda de un país en estos cuatro términos suma un porcentaje de $100 \%$, debido a que este país figura en las cinco naciones más relevantes para el término, por ejemplo, México es un país con mayor interés en todos los términos. No obstante Marruecos es un país interesado en FMPT, SECTUR Federal y SECTUR CDMX, sin embargo, no llega al 100\% ya que no está entre los países más interesados en el CPTM. Este mismo proceso aplica para todas las categorías.

Posteriormente se determinaron las palabras clave relacionadas con el turismo y con la marca CDMX, para lo cual hubo un respaldo en las plataformas SEM rush y Pro similar WEB, ya que al buscar el término CDMX, estos sitios plasmaron los términos con mayor relación a este a nivel mundial, se incluyeron solo los cuatro términos clave más relevantes con la finalidad de dejar el mismo número de elementos en cada categoría, estos son tourism, mexicocitytourism, Mexicocity y CDMX. Al igual que en la categoría anterior, se plasmaron a los cinco países más interesados en cada uno de los términos y se asignó un valor en términos de porcentaje.

Por último, se definieron con base en Google, las páginas con mayor número de visitas a nivel mundial relacionadas con el área turística en México. Ante ello se determinó que las páginas con mayores búsquedas relacionadas con la actividad son CDMX travel, VisitMexico, TuribusMexico y Tripadvisor, para ello se buscó en Google la palabra CDMX tourism y se obtuvieron estas páginas como las referencias principales. Se aplicó el mismo procedimiento, de tal forma se analizó a los países con mayor interés en la actividad. Una vez construida una tabla 
con los porcentajes de búsqueda de estos organismos, términos y páginas con su respectivo cruce con los países más interesados en estos, se aplica el análisis de redes la cual se considera un análisis cuantitativo para medir la centralidad por con apoyo del software Pajek:

"éste es un software desarrollado por un grupo de investigadores de la Facultad de Ciencias de la Universidad de Eslovenia. Cabe señalar que desde su presentación a mediados de los años 1990s, ha sido considerado por la comunidad como uno de los mejores programas del área" (Ruiz y Rusell, 2016, p.17).

Se modelan las redes, ya que estas son un modelo que permite abstraer los datos, plasmando con ello "una imagen, un dibujo o una gráfica de la realidad" (Render, 2012, p.4). Las redes sociales son "un conjunto finito de actores y de relaciones definidas entre ellos" (Vivas, 2001, p. 93), por ello en estas con base en Wasserman y Faust (1994) pueden estudiarse los actores, los recursos que intercambian, los atributos de la relación social y la intensidad de la fuerza del vínculo creado.

La red se considera un modelo matemático que permite la representación de los valores y con ello se ilustran las relaciones en el sistema. Respecto a la centralidad, la misma hace referencia a los vínculos que un nodo puede tener con otros en la red, por ello el número muestra la concentración del interés entre los diversos términos analizados. "La centralidad es un importante atributo estructural de las redes sociales. Todos aceptan que está relacionada en un alto grado con otras importantes propiedades y procesos grupales" (Freeman, 2000, p.132). De tal forma, por medio del análisis de la centralidad se logra una representación visual y matemática de la forma en que se interconectan los organismos logrando una interpretación visual del fenómeno.

Figura 1. Metodología

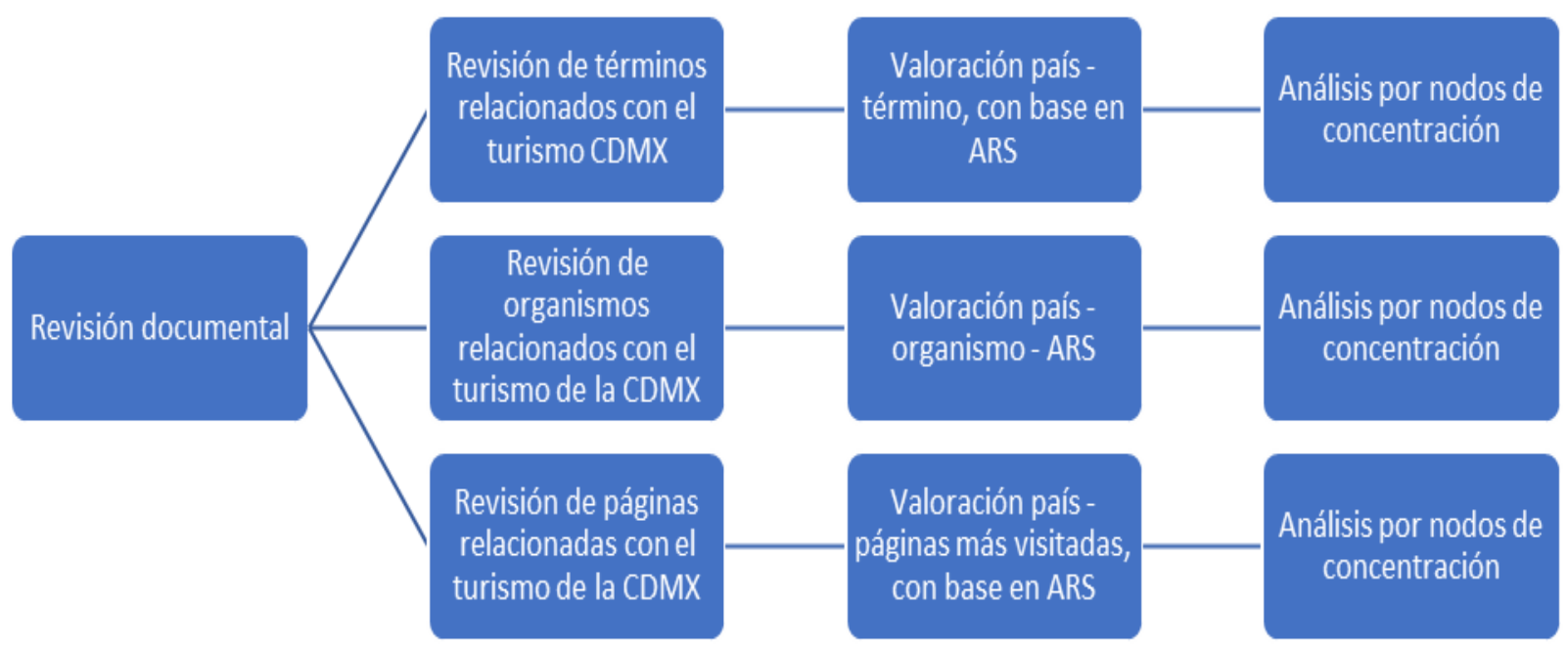

Elaboración propia. 


\section{RESULTADOS}

El Análisis de Redes Sociales, permite un análisis del comportamiento por acceso en países interesados a la Web con palabras clave, así como de la intervención de organismos estatales. En el Anexo 1 se plasma una matriz con el eje horizontal, en el que aparecen los países (19 países participantes) con mayor interés en las diferentes categorías analizadas respecto a la actividad turística de la Ciudad de México. En el eje vertical se encuentran en una primera sección los organismos, en la segunda 4 términos clave y en la tercera las 4 páginas más visitadas. Los cruces con los respectivos porcentajes son referentes a la actividad.

Mientras el Anexo 2 se encuentra la centralidad analizada con apoyo de Pajek, se enlistan cuatro categorías, entre ello se destaca que el organismo más buscado a nivel internacional es el CPTM con una centralidad de .5312, la palabra clave con mayores búsquedas es Mexicocity con un índice .1700, la página con mayor búsqueda es VisitMexico la cual sobresale con un indicador de .8207 y el país con mayor interés en los distintos organismos, palabras y páginas de la Ciudad de México es Estados Unidos, el cual se ubica con un índice de .5783.

La Figura 2 ilustra los nodos con mayor centralidad en el sistema. De acuerdo con la representación gráfica los términos CPTM, Mexicocity, la página VisitMexico y el país Estados Unidos, son los puntos más relevantes en el sistema confirmado con el tamaño de las figuras. Estos puntos gráficos más adelante se confirman con base en las mediciones de centralidad hechas con apoyo del software Pajek.

Figura 2. Red de términos de búsqueda y países con mayor interés en el turismo de la Ciudad de México

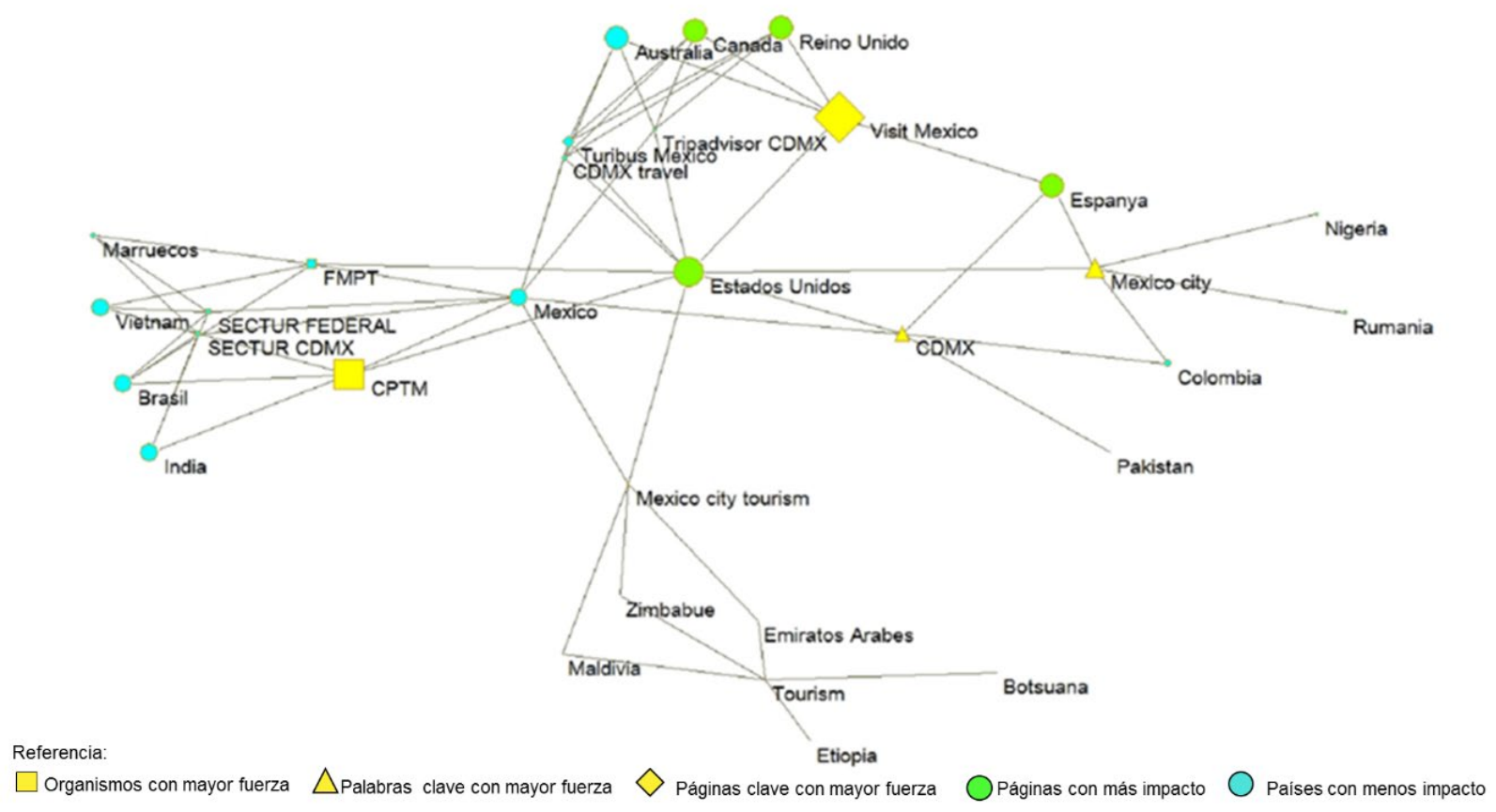

Elaboración propia con base en Pajek. 
La Figura 3 muestra los organismos, términos y páginas con mayores búsquedas, pero al mismo tiempo se muestra la relación de estos con los países de mayor interés en el sistema. Se enfatizan estas relaciones gráficamente con las líneas entre los nodos.

Figura 3. Red de términos de búsqueda y su relación con los países con mayor interés en el turismo de la Ciudad de México

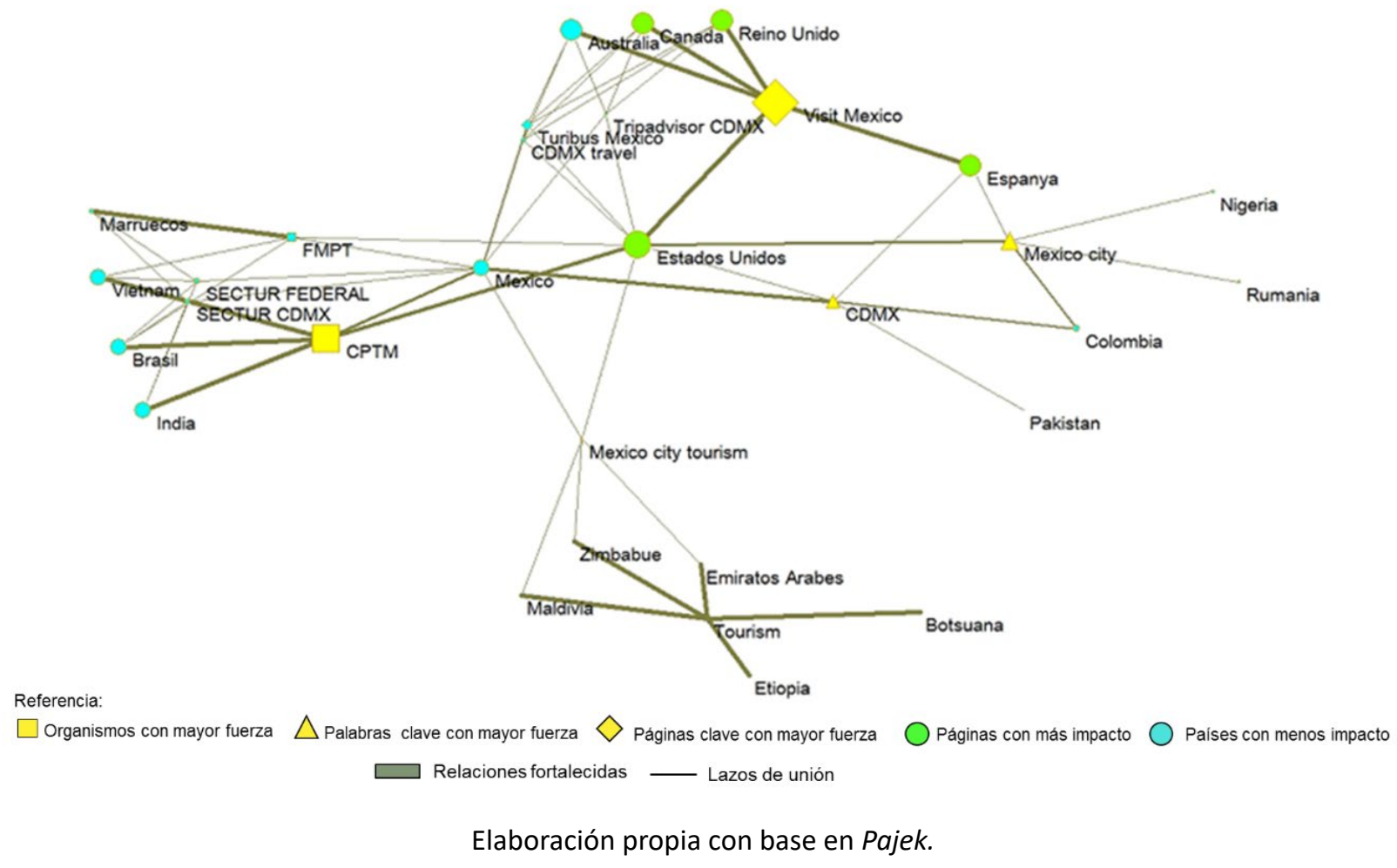

\section{DISCUSIÓN DE RESULTADOS}

La globalización impone a los productos y servicios la necesidad de estar presente no solo en su país de origen, sino de hacer frente a la competencia y demanda de cualquier parte del mundo. En el caso de los destinos turísticos esto no es la excepción, ya que la Ciudad de México como producto busca atraer no solo a los viajeros nacionales, sino el capital internacional, dado el ingreso de divisas y por ende los beneficios que ello trae consigo. Para lograrlo se destinan una gran cantidad de recursos para el desarrollo de material en cuanto a páginas Web y publicidad a través de diversos medios e internet. Al medir en este caso desde internet como se observa la recepción de estos recursos, es visible que hay países en los que hay un interés por el destino, ya que en general hay búsquedas hacia diversos organismos, términos y páginas relacionados con la actividad turística.

El ARS: “[...] como una técnica de análisis estructural, es sin duda una alternativa metodológica en el estudio del turismo, reconociendo la naturaleza relacional de gran parte de sus actividades económicas" (Zermeño Cuevas- Contreras, y Zizaldra- Hernández, 2018, p.94). Esta técnica permite el análisis de la centralidad vista en el Anexo 2, la cual plasma el CPTM con 
un índice de .5312 -(Figura 3, concentración 2) organismo en el que hay mayor interés a nivel internacional -, ya que el FMPT que es el segundo organismo con mayor interés del público se encuentra muy lejano aún con un grado de centralidad de .0552, de tal forma es importante que este Consejo tenga mensajes claros que apoyen el desarrollo de la actividad y puedan impulsar la misma.

En cuanto a los términos buscados se observa un mayor interés en Mexicocity (Figura 3) con un índice de centralidad de .17, frente a una centralidad de .0982 de CDMX. Resulta significativo este análisis ya que acorde a la página del FMPT en 2014 se lanzó la misma como la marca de la Ciudad, de tal forma puede observarse que aún esta requiere esfuerzos para su consolidación, viéndose distante con respecto a marcas reconocidas como son New York o París. Por lo anterior, es necesario dar un gran peso a esto ya que "la estrategia de marca país se ha convertido en un propósito de los gobiernos por mejorar su reputación en mercados internacionales. El resultado final de aplicar la estrategia de marca país no es sólo enmendar la imagen de un país, sino cambiar la percepción de los visitantes (turistas e inversionistas) a través de una experiencia positiva sobre un destino" (Echeverri, Estay-Niculcar, Herrera, y Santamaría, Juliana, 2013, p. 1121).

Figura 4. Análisis de la Red por nodos de concentración de interés en CDMX

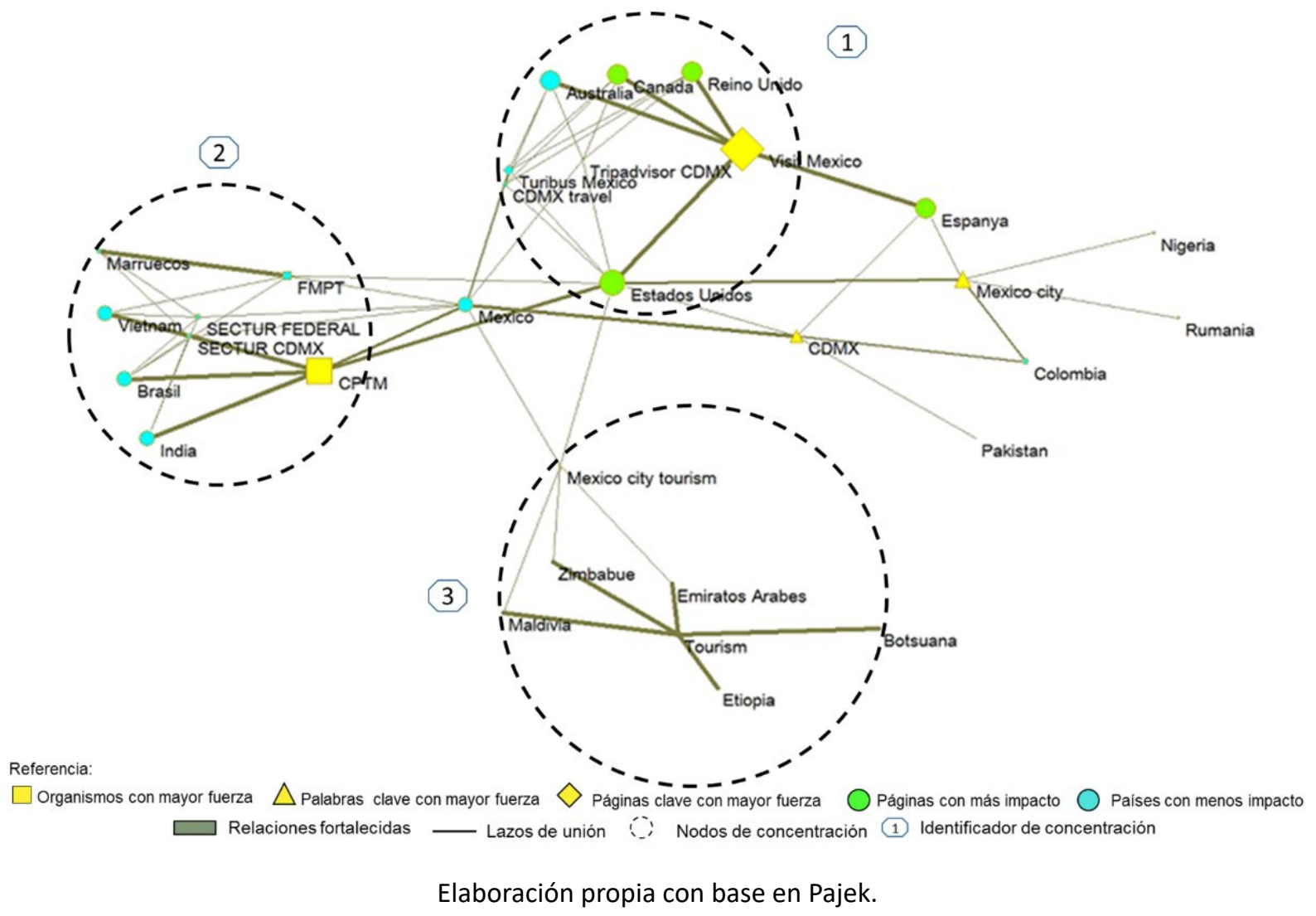

Respecto a la página con mayores visitas se encuentra VisitMexico (Figura 4, concentración 1) con un grado de centralidad de .8207 , lo cual es muy relevante, ya que esta página 
al exhibir diversos destinos mexicanos, puede ser un exponente clave de las posibilidades de la Ciudad de México y de la diversidad de actividades que hay en la Ciudad. La página del Turibus es también de interés en el entorno internacional, ya que ofrece un servicio similar al de principales capitales. El transporte de turismo facilita el desplazamiento de los visitantes en ciudad, brindando visita a lugares iconos, como lo es el paquete a las luchas libres. Es un servicio de transporte aun poco concurrido, y que puede ser pauta de difusión. En relación con: “El CPTM [Figura 3, concentración 2] fortalece la actividad turística con la marca México, a través de la cual constantemente identifica las oportunidades de mejora de la imagen en el mercado nacional e internacional como destino turístico (Cuevas-Contreras et al, 2017, p.175)".

En cuanto a los países no es de sorprender que el país vecino (Figura 3, concentración 1), dada su cercanía geográfica se interese en la nación mexicana y especialmente en la Ciudad de México, ya que es un lugar en el que pueden realizarse diversos tipos de turismo y actividades que pueden estar relacionadas con los negocios. Debe aprovecharse este interés observado por los estadounidenses con un índice de .5783 , ya que son un mercado muy grande, con una cercanía y facilidad de viaje, derivado también de la devaluación de la moneda mexicana. Si bien:

[...] las metodologías automatizadas de evaluación de experiencia de visita a destinos turísticos actuales aún no permiten formular inferencias estadísticas; su incipiente nivel de desarrollo, experimental y exploratorio, no se considera suficientemente evolucionado para aportar recomendaciones o soluciones a empresarios o encargados de la gestión de destinos turísticos, por el momento (Amaya, Magaña y Ochoa, 2017, p.301).

Si bien este aspecto es contradictorio para una gran cantidad de actividades económicas, esta devaluación le favorece al turismo, ya que el turista internacional eleva su capacidad de compra y por ende México puede ser más atractiva que otros destinos internacionales, por lo accesibilidad de sus precios.

En cuanto a la Figura 4, concentración 3, el lazo de unión es Estados Unidos y México, a través de México citytourism. Es una concentración de países (Zimbabue, Maldivia, y Emiratos Árabes) heterogéneos, que sin embargo guardan un interés mediante la revisión de la Web, aunque en el sistema aparecen Etiopía y Botsuana, estos son visibles en el sistema dado su interés en el turismo en general, pero no directamente con la oferta mexicana. Este interés plasmado en la web, muestra la capacidad de atracción del destino mexicano como uno de los 10 países más visitados, de acuerdo con la UNWTO. De manera que, su disposición queda como una relación fortalecida ante el punto de unión de Tourism.

\section{CONCLUSIONES}

El método de investigación a través de la cual se aborda el fenómeno determina en gran medida los datos obtenidos y la interpretación de estos. El conocer en una primera etapa como el Smart data plasma de manera puntual el resultado procesado de grandes volúmenes de información, soporta los resultados después plasmados a través de ARS, ya que si bien, por medio de la Web es posible proporcionar información clave del manejo de diversos términos 
en el entorno digital, el medir la centralidad, permite dar una visión puntual, de cómo se comporta la audiencia en torno a los esfuerzos realizados, lo que da la oportunidad de tener una visión holística del fenómeno en las redes, observando así como se vinculan los resultados de la comunicación publicitaria. Aunado a ello la construcción de este modelo es matemático, lo cual da validez a una representación visual.

Como contribución la aplicación de un método bajo el cual no se había explorado la actividad, resulta importante, ya que plasma la situación en la que se encuentra el destino en un mundo altamente competitivo, que demanda que las diversas económicas del país busquen la atracción del capital internacional para la mejora de la calidad de vida de sus ciudadanos. Este abordaje lo hace desde una perspectiva matemática que apoya un análisis puntual.

Se evidencia, la influencia de la globalización en los primeros tres lustros del siglo XXI ha sido fundamentales en los cambios de los medios de comunicación. La actividad turística se ha visto estos cambios. La investigación realizada con base en la investigación documental y la aplicación del análisis de redes a los países con mayor interés en los organismos, términos y páginas que exhiben a la Ciudad de México, muestra que hay en general una nación que destaca del resto en cuanto al interés en el turismo de la Ciudad de México, el cual es Estados Unidos. De tal forma debe aprovecharse la cercanía geográfica con el destino a través de las diversas formas existentes de comunicación tanto digital como tradicional para llegar con mayor fuerza a este mercado, ya que de acuerdo con Sectur en 2017, el país recibió un 10.6 millones de visitantes estadounidenses, lo cual implica un crecimiento del 9.6 por ciento en comparación con el 2017 (Sectur, 2018). Debe considerarse que otros países con un interés en el destino son: España, Canadá, Reino Unido y Australia.

Es vital el aprovechamiento de diversos factores que pueden favorecer la relación comercial como lo son el interés en el destino, la cercanía geográfica, la conectividad aérea y la devaluación de la moneda mexicana, ya que estos factores en definitiva pueden tener un gran impacto para empujar esta relación comercial. Asimismo, debe considerarse la coyuntura de potencializar el uso del término CDMX, ya que "en la actualidad una marca se constituye en un valor agregado cuando las percepciones sobre un país, región o ciudad son positivas, lo que propicia una impresión favorable de las marcas de productos fabricados en ese lugar y potencia toda una serie de actividades" (Calvento y Ochoteco, 2009, p.65). Por ello debe trabajarse en conjunto con la finalidad de que haya un empuje en este sentido que pueda llevar con los años a lograr una percepción positiva de la ciudad y un mensaje que transmita la dimensión de lo que es la Ciudad de México.

Finalmente, las páginas deVisitMexico y el CPTM, deben aprovecharse también dado el gran interés que se observa en el mercado internacional con respecto a estos organismos, demostrando con ello como pueden contribuir en buena medida a atraer a los turistas internacionales y con ello favorecer el desarrollo de la actividad en el país.

Como limitantes debe reconocerse que el estudio realizado solo plasma los datos más relevantes, sin embargo, hay muchos otros términos, países y organismos que podrían considerarse para un análisis más amplio. Aunado a ello debe tomarse en cuenta, como una nueva línea de investigación, la valoración de la forma en que la imagen administrativa de la CDMX 
a partir del ingreso del nuevo gobierno, puede influir en la conformación de identidad de la Ciudad de México y en la percepción del mercado nacional e internacional ${ }^{1}$.

\section{BIBLIOGRAFÍA}

Amaya, C. M.; Magaña, P. y Ochoa, I. (2017). Evaluación de destinos turísticos mediante la tecnología de la ciencia de datos. Estudios y Perspectivas en Turismo, 26, 286-305.

Amon, D., Arcides, P., Roso, A. y Veríssimo, M. (2014). Propaganda, publicidad y opinión pública. Dimensiones éticas. Subjetividad y Procesos Cognitivos. 18 (2), 33-52.

Brida, J., Lanzilotta, B., Pereyra, J. y Pizzolon, F. (2013). El turismo como factor del crecimiento económico: un estudio comparativo de los países del MERCOSUR. Revista de Economía Mundial, 34, 75-96.

Calvento, M. y Ochoteco, M. (2009). Una aproximación a la construcción de marca-ciudad como estrategia de inserción nacional e internacional. Economía, Sociedad y Territorio, IX, 29, 59-87.

Camargo-Vega, J., Camargo-Ortega, J. y Joyanes, L. (2015). Conociendo BIG DATA. Facultad de Ingeniería, 24 (38), 63-77.

Conde, N. (2013). Análisis de la llegada de turistas internacionales. Investigación Administrativa, 111, 20-34.

Cuevas, T.; Zizaldra, I. y Carrillo, L. G. (2017). Turismo en la gestión pública de México. Desde finales del siglo XX y albores del XXI. En Noemí Wallingre. (Eds.) Desarrollo del turismo en América Latina: fases, enfoques e internacionalización, (pp. 170-196). Bernal: Universidad Nacional de Quilmes.

Datatur. Indicadores macroeconómicos. Recuperado de: http://www.datatur.sectur.gob.mx/ SitePages/IndicadoresMacroeconomicos.aspx

Datatur. Resultados de la Actividad Turística 2018. Recuperado de: https://www.datatur.sectur. gob.mx/RAT/RAT-2018-12(ES).pdf

Echeverri, L., Estay, C., Herrera, C. y Santamaría, J. (2013). Desarrollo de marca país y El caso de estudio de México. Estudios y Perspectivas en Turismo, 22 (6), 1121-1139.

Freeman, L. (2000). Política y sociedad. Madrid: University of California at Irvine.

Gómez, B. y Benito, C. (2014). Presente de la Comunicación organizacional en la PyME Española. Razón y Palabra, 18 (86).

Goyzueta, S. (2015). BIG DATA Marketing: una aproximación. PERSPECTIVAS, 35, 147-158.

Hernández, E., Duque, N. y Moreno, J. (2017). BIG DATA: una exploración de investigaciones, tecnologías y casos de aplicación. Tecno Lógicas, 20, 39.

Hootsuite. (2018). Digital in 2018. Recuperado de: file:///C:/Users/sasel/AppData/Local/ Packages/Microsoft.MicrosoftEdge_8wekyb3d8bbwe/TempState/Downloads/Digitalin-2018-001-Global-Overview-Report-v1.02-L.pdf

INEGI. (2019). Establecimiento de salud en establecimientos particulares. Recuperado de: https://www.inegi.org.mx/sistemas/olap/consulta/General ver4/MDXQueryDatos. asp?\#Regreso\&c=33418

1. Investigación realizada gracias al apoyo UNAM - DGAPA. 
Jova, R., Bradshaw, A. y Despaigne, H. (2015). Streaming de archivos multimedia desde bases de datos. Revista Cubana de Ciencias Informáticas, 9 (2), 1-13.

Ley General de Turismo. Nueva Ley publicada en el Diario Oficial de la Federación, México, 17 de junio de 2009.

Ley de Turismo del Distrito Federal. Gaceta Oficial del Distrito Federal, México, 10 de agosto de 2013.

Luque, A., Zayas, B. y Caro, J. L. (2015). Los Destinos Turísticos Inteligentes en el marco de la Inteligencia Territorial: conflictos y oportunidades. Investigaciones Turísticas, 10, 1-25.

Morillo, M. (2011). Turismo y producto turístico. Evolución, conceptos, componentes y clasificación. Visión Gerencial, 1, 135-138.

Orgaz, F. y Moral, S. (2016). El turismo como motor potencial para el desarrollo económico de zonas fronterizas en vías de desarrollo. Un estudio de caso. El Periplo Sustentable, vol. 31.

Ortiz, M., Aguilar, L., y Giraldo, L. (2015). Los desafíos del marketing en la era del bigdata. ECiencias de la Información, 6, 1, 1-30.

Plasencia, L. y Anías, C. (2017). Arquitectura referencial de BIG DATA para la gestión de las telecomunicaciones. Ingeniare. Revista Chilena de Ingeniería, 25 (4), 566-577.

Render, B. (2012). Métodos cuantitativos para los negocios. México: Pearson.

Robbins, S. y Coulter, M. (2014). Administración. México: Pearson

Ruiz, A. y Russell, J. (2016). La estructura del sistema científico de México a finales del siglo XX: una visión a nivel de instituciones. Redes. Revista hispana para el análisis de redes sociales, [S.I.], 27, 2, 11-32

Sobrino, L. (2002). Competitividad y ventajas competitivas: revisión teórica y ejercicio de aplicación a 30 ciudades de México. Estudios Demográficos y Urbanos, 50, 311-361.

SECTUR. (2018). Conectividad aérea, factor clave en el flujo turístico de Estados Unidos a México: De la Madrid. Recuperado de: https://www.gob.mx/sectur/prensa/ conectividad-aerea-factor-clave-en-el-flujo-turistico-de-estados-unidos-a-mexico-de-la-madrid?idiom $=$ es

UNWTO. (2019). UNWTO. World Tourism Organization. Recuperado de: http://www2.unwto. org/

UNWTO. (2019). Glosario de términos de turismo. Recuperado de: https://www.unwto.org/ es/glosario-terminos-turisticos

Vivas, J. (2001). Análisis de redes sociales y procesos de influencias en la toma de decisión grupal interdisciplinaria. Centro Interamericano de Investigaciones Psicológicas y Ciencias Afines Argentina. 18, 1, 87-113.

Wasserman, S. y Faust, K. (1994). Social Network Analysis. Methods and Applications. Cambridge: Cambridge University Press.

Zermeño, S. G., Cuevas, T. y Zizaldra, I. (2018). Reticularidad e innovación en actores y stakeholders de turismo de salud: caso de competitividad en Los Algodones, Baja California. Noesis, 27, 53-2, 78-97

Zúñiga, E. (2010). La comunicación organizacional y su importancia para las escuelas de arte universitarias: Propuesta para la construcción de conocimiento organizacional colectivo desde una intervención educativa. Revista Educación, 34 (1), 73-81. 


\section{ANEXOS}

Anexo1: Tabla 1. Países con mayor interés en la WEB en el turismo de la CDMX

\begin{tabular}{|c|c|c|c|c|c|c|c|c|c|c|c|c|c|c|c|c|c|c|c|}
\hline Organismos & 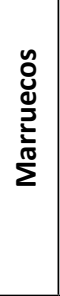 & 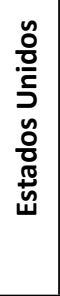 & : & $\begin{array}{l}\frac{\pi}{0} \\
\frac{0}{0} \\
\frac{0}{0}\end{array}$ & $\begin{array}{l}\overline{\bar{n}} \\
\overline{\overline{0}} \\
\overline{\bar{\omega}}\end{array}$ & 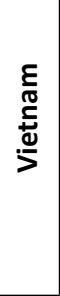 & $\begin{array}{l}\frac{\pi}{0} \\
\frac{\pi}{\pi} \\
\frac{\pi}{10} \\
0\end{array}$ & $\stackrel{\text { 음 }}{\underline{\underline{C}}}$ & 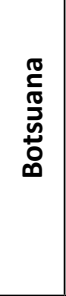 & 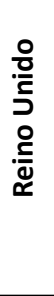 & 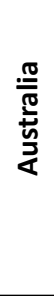 & 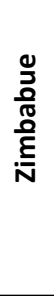 & $\begin{array}{l}\frac{\pi}{0} \\
\frac{0}{\circ} \\
\stackrel{\mathcal{H}}{2}\end{array}$ & $\frac{\frac{\pi}{2}}{\frac{\pi}{0}}$ & 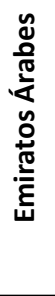 & 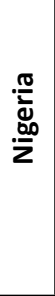 & 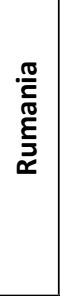 & 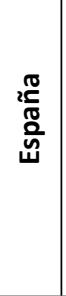 & 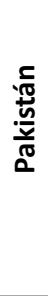 \\
\hline FMPT & 98 & 16 & 5 & 0 & 1 & 1 & 0 & 0 & 0 & 0 & 0 & 0 & 0 & 0 & 0 & 0 & 0 & 0 & 0 \\
\hline SECTUR FEDERAL & 1 & 0 & 17 & 0 & 1 & 1 & 0 & 1 & 0 & 0 & 0 & 0 & 0 & 0 & 0 & 0 & 0 & 0 & 0 \\
\hline SECTUR CDMX & 1 & 0 & 19 & 0 & 1 & 1 & 0 & 1 & 0 & 0 & 0 & 0 & 0 & 0 & 0 & 0 & 0 & 0 & 0 \\
\hline CPTM & 0 & 84 & 59 & 0 & 97 & 97 & 0 & 98 & 0 & 0 & 0 & 0 & 0 & 0 & 0 & 0 & 0 & 0 & 0 \\
\hline Términos clave & & & & & & & & & & & & & & & & & & & \\
\hline Tourism & 0 & 0 & 0 & 0 & 0 & 0 & 0 & 0 & 100 & 0 & 0 & 99 & 1.0 & 99 & 97 & 0 & 0 & 0 & 0 \\
\hline Mexicocitytourism & 0 & 1 & 1 & 0 & 0 & 0 & 0 & 0 & 0 & 0 & 0 & 1 & 0 & 1 & 1 & 0 & 0 & 0 & 0 \\
\hline Mexicocity & 0 & 57 & 0 & 36 & 0 & 0 & 0 & 0 & 0 & 0 & 0 & 0 & 0 & 0 & 0 & 14 & 10 & 14 & 0 \\
\hline CDMX & 0 & 3 & 90 & 33 & 0 & 0 & 0 & 0 & 0 & 0 & 0 & 0 & 0 & 0 & 0 & 0 & 0 & 9 & 1 \\
\hline $\begin{array}{l}\text { Páginas más } \\
\text { visitadas }\end{array}$ & & & & & & & & & & & & & & & & & & & \\
\hline CDMX travel & 0 & 1 & 4 & 0 & 0 & 0 & 1 & 0 & 0 & 1 & 1 & 0 & 0 & 0 & 0 & 0 & 0 & 0 & 0 \\
\hline VisitMexico & 0 & 97 & 0 & 0 & 0 & 0 & 97 & 0 & 0 & 97 & 97 & 0 & 0 & 0 & 0 & 0 & 0 & 100 & 0 \\
\hline TuribusMexico & 0 & 1 & 46 & 0 & 0 & 0 & 1 & 0 & 0 & 1 & 1 & 0 & 0 & 0 & 0 & 0 & 0 & 0 & 0 \\
\hline Tripadvisor CDMX & 0 & 1 & 1 & 0 & 0 & 0 & 1 & 0 & 0 & 1 & 1 & 0 & 0 & 0 & 0 & 0 & 0 & 0 & 0 \\
\hline
\end{tabular}

Elaboración propia. 
Anexo 2: Tabla 2. Centralidad analizada con apoyo de Pajek

\begin{tabular}{|l|l|}
\hline \multicolumn{2}{|c|}{ Organismos } \\
\hline CPTM & 0.5312 \\
\hline FMPT & 0.0552 \\
\hline $\begin{array}{l}\text { SECTUR } \\
\text { CDMX }\end{array}$ & 0.0179 \\
\hline $\begin{array}{l}\text { SECTUR } \\
\text { FEDERAL }\end{array}$ & 0.0164 \\
\hline & \\
\hline & \\
\hline & \\
\hline & \\
\hline & \\
\hline & \\
\hline & \\
\hline & \\
\hline & \\
\hline & \\
\hline & \\
\hline & \\
\hline & \\
\hline & \\
\hline & \\
\hline & \\
\hline & \\
\hline & \\
\hline & \\
\hline & \\
\hline & \\
\hline & \\
\hline & \\
\hline & \\
\hline & \\
\hline & \\
\hline & \\
\hline
\end{tabular}

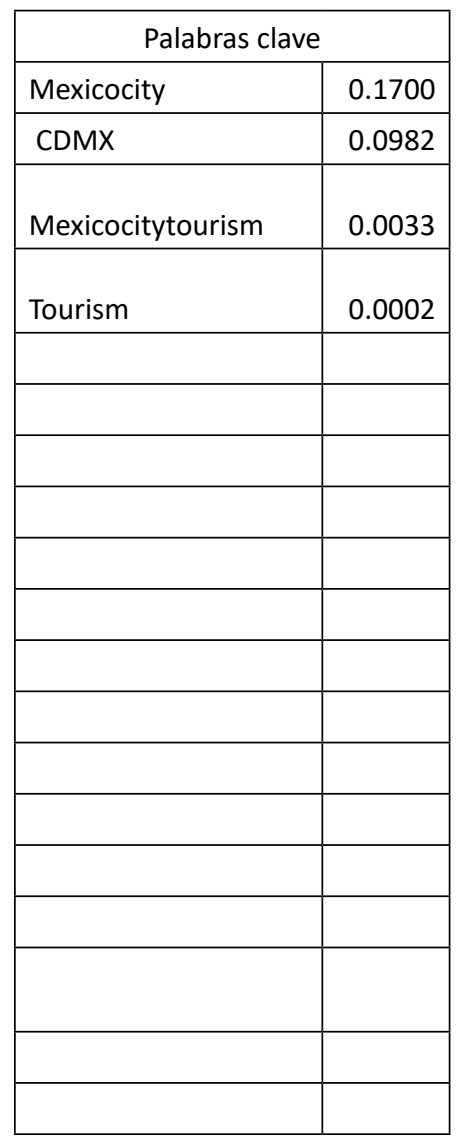

\begin{tabular}{|c|c|}
\hline \multicolumn{2}{|c|}{ Páginas } \\
\hline VisitMexico & 0.8207 \\
\hline TuribusMexico & 0.0432 \\
\hline CDMX travel & 0.0100 \\
\hline $\begin{array}{l}\text { Tripadvisor } \\
\text { CDMX }\end{array}$ & 0.0076 \\
\hline & \\
\hline & \\
\hline & \\
\hline & \\
\hline & \\
\hline & \\
\hline & \\
\hline & \\
\hline & \\
\hline & \\
\hline & \\
\hline & \\
\hline & \\
\hline & \\
\hline & \\
\hline
\end{tabular}

\begin{tabular}{|l|l|}
\hline \multicolumn{2}{|c|}{ Países } \\
\hline Estados Unidos & 0.5783 \\
\hline España & 0.3651 \\
\hline & 0.3409 \\
\hline Canadá & 0.3409 \\
\hline Reino Unido & 0.3409 \\
\hline Australia & 0.2229 \\
\hline India & 0.2209 \\
\hline Brasil & 0.2209 \\
\hline Vietnam & 0.1845 \\
\hline México & 0.0401 \\
\hline Colombia & 0.0233 \\
\hline Marruecos & 0.0102 \\
\hline Nigeria & 0.0073 \\
\hline Rumania & 0.0004 \\
\hline Pakistán & 0.0001 \\
\hline Zimbabue & 0.0001 \\
\hline Maldivia & 0.0001 \\
\hline Emiratos & 0.0001 \\
\hline Árabes & 0.0001 \\
\hline Etiopía & \\
\hline
\end{tabular}

Elaboración propia. 